\title{
Fonksiyon Yaklaşımı Probleminde Esnek Küçük-Dünya Ağlarının Topolojik Değişiminin Performansa Etkisi
}

\author{
Okan ERKAYMAZ \\ Zonguldak Bülent Ecevit Üniversitesi, Mühendislik Fakültesi, Bilgisayar Mühendisliği Bölümü, Zonguldak \\ Türkiye \\ *okanerkaymaz@yahoo.com
}

Received/Geliş: 30.08 .2020

Accepted/Kabul: 12.09 .2020

\begin{abstract}
Öz: İleri yönlü yapay sinir ağları performansı topolojiyle doğrudan ilişkilidir. Son yıllarda, topolojik değişikliklerle ağ performansının artırılması üzerine yapılan çalışmalar çok önemli bir süreç haline gelmiştir. Bu nedenle, nöronlar arası sinaptik bağlantıların değiştirildiği bir yaklaşım olan küçük-dünya ağ modeli gelecek çalışmalar için önemli bir yere sahiptir. Bu çalışmada, fonksiyon yaklaşımı problemleri için esnek küçük-dünya ağ modelinin katmansal ve nöronal değişimlerle performansının nasıl değiştiği araştırılmıştır. Elde edilen sonuçlar gösterdi ki, geleneksel esnek yapay sinir ağı topolojisine göre esnek küçük-dünya ağının performansı çok daha fazla iyileştirdiği ve katman sayısının artırılmasının ağ performansına ciddi katkı sağladığı gözlemlenmiştir. Diğer taraftan, katman sayısı ile birlikte gizli katman nöron sayısının artırıldıkça performansa negatif etki ettiği görülmüştür. Bu bağlamda, çoklu gizli katmana sahip esnek küçük-dünya ağlarının fonksiyon yaklaşımı probleminin çözümünde pozitif etki ettiği gösterilmiş ve özellikle bu modelin gelecekte derin nöron ağları performansının geliştirilmesinde anahtar rol oynayacağı vurgulanmıştır.
\end{abstract}

Anahtar Kelimeler: Newman-Watts algoritması, esnek küçük-dünya ağı, fonksiyon yaklaşımı, bağlantı yenileme.

\section{Effect of Topological Change of Resilient Small-World Networks on Performance in the Function Approach Problem}

\begin{abstract}
Performance of Feed forward neural networks is directly related to topology. In recent years, studies on improving performance of network with topological changes have become a very important process. Therefore, the small-world network model, an approach in which synaptic connections between neurons are altered, has an important place for future studies. In this study, how the performance of the resilient small-world network model changes with layered and neuronal revisions has been investigated for function approach problems. The results show that the resilient small-world network improves the performance much more than the traditional resilient artificial neural network topology and it has been observed that increasing the number of layers significantly contributes to the network performance. On the other hand, It has been observed that when the number of hidden layer neurons is increased with the number of layers, the performance has a negative effect. In this context, it has been shown that resilient small-world networks with multiple hidden layers have a positive effect on the solution of the function approach problem and also it is emphasized that this model will play a key role in improving the performance of deep neuron networks in the future.
\end{abstract}

Keywords: Newman-Watts algorithm, resilient small-world network, function approach problem, rewiring.

\section{Giriş}

Günümüzde, Yapay zekâ insanlık için en önemli bir destekleyici bilgi kaynağı olarak görülmektedir. Yapay zekâ, insanın problem çözerken kullandığı düşünce kurgusunu modelleyerek 
matematiksel benzetim yapan bir algoritmadan ibarettir. Böylece, doğru bilgi kaynağı ile hata riski daha az olan karar destek sistemleri ortaya konulabilmektedir [1]. Son yıllarda, bu alana ilgilinin artmasıyla, pek çok yapay zekâ modeli önerilmiştir. Bunlar; Yapay sinir ağları (YSA), Bulanık mantık küme teorisi (BM), Uzman sistemler (US), Genetik algoritmalar (GA), Destek Vektör Makineleri (DVM) vb. gibi. Önerilen modellerden, yüksek genelleme yeteneğinden dolayı YSA yaklaşımı, çok sık tercih edilen ve pek çok endüstriyel alana uygulanan bir araç olmuştur. YSA, özelikle insan beyninin karmaşık problemleri çözerken veya bilgiyi saklarken kullandığı nöronal davranışı betimlemektedir [1]. Topolojik olarak YSA, yapay nöronlar ve katmansal yapılar arası ileri ve geri yönlü oluşturulan bağlantılardan oluşmaktadır. Bu mimari yapı, beynin paralel veri işleyebilme yeteneğini YSA nöronlarına kazandırmaktadır. Biyolojik nöron ağlarının veriyi kodlayarak hücreden hücreye aktarmasına bezer şekilde, YSA mimarisi de katmanlar arası nöronların birbirleriyle kurdukları bağlantıların gücünü değiştirerek bilgiyi kodlar. $\mathrm{Bu}$ nedenle, bağlantıların sinaptik gücünün değiștirilmesi süreci yapay öğrenme olarak kabul edilmektedir [2-4]. Öğrenme sürecinin modellenmesinde özellikle İleri Yönlü YSA ağlarında; geri yayılım (Backpropagation, BP), Esnek geri yayılım (Resilient Back-propagation, Rprop)[4] ve Levenberg Marquard(LM) algoritmaları en bilinen algoritmalardır. Bunlardan Rprop ve LM algoritmaları geri yayılım algoritmasının yavaş öğrenme ve aşırı öğrenme sorunlarına çözüm olmuştur [5-7].

YSA topolojisinde öğrenme, algoritma türü ve topolojik değişikliklere bağlıdır. Özellikle son yıllarda, klasik YSA mimarilerinin yanında derin YSA mimarilerinde de görüldüğü gibi öğrenme sürecini etkileyen en önemli faktörlerden biri olan topolojik değişimler önemli bir araştırma konusu olmuştur. Bu araştırmalar özellikle İleri Yönlü YSA modellerini temel alarak ilerlemektedir. İleri yönlü YSA modelinde, topolojik değişiklikler daha çok katmansal yapıda nöronlar arası sinaptik bağlantı farklılıklarıdır [2-4].

Biyolojik nöron ağları üzerine yapılan araştırmalar, biyolojik nöronun bilgiyi elektriksel veya kimyasal kodlaması ve iletimi üzerine yoğunlaşmıştır [8-9]. Bu süreçte, küçük-dünya (SmallWorld, SW) [10-13] ve ölçeksiz (Scale-free, SF) [14] ağ topolojileri biyolojik nöron ağı davranışını modelleyen iki önemli matematiksel model olarak önerilmiştir. Diğer taraftan, önerilen bu ağ modelleri YSA topolojilerine yeni bir ş̧ık tutmuştur. YSA topolojilerinde, Simard ve arkadaşları [15], İleri yönlü YSA topolojisinde SW ağı davranışını incelemişler ve performansı iyileştirdiğini vurgulamışlardır. Aynı zamanda, Shuzhong ve arkadaşları [16], ileri yönlü YSA ağlarında SW davranışının öğrenme hatasını ve öğrenme zamanını azalttığını göstermişlerdir. Erkaymaz ve arkadaşları [4,17], ileri Yönlü YSA mimarisinde Watt-strogatz yaklaşımının daha geniş ölçekli bağlantı yenilemesiyle SW etkisinin gözlemlenebileceğini göstermiştir. Ek olarak, Erkaymaz ve arkadaşları [18-19], literatürde ilk defa, Newman-Watts SW yaklaşımının ileri Yönlü YSA modellerinde performansı artırdığını göstermişlerdir. Diğer taraftan, Erkaymaz, 2020 [20], Newman-Watts temelli İleri yönlü YSA topolojisinde esnek geri yayılım öğrenme algoritmasının kullanılabilirliğini göstererek Esnek küçük-dünya ağ modelini önermiş ve önerilen modelin performansının diğer modellerden daha iyi olduğunu ortaya koymuştur.

Bu çalışmada, fonksiyon yaklaşımı problemi için, Erkaymaz [20] tarafından önerilen Esnek küçükdünya ağlarıyla çok katmanlı topolojiler oluşturulmuş ve elde edilen topolojilerin performans analizleri gerçekleştirilmiştir.

\section{Esnek Küçük-Dünya Ăğı Modeli}

Esnek küçük-dünya ă̆ modeli, düzenli ağ olarak kabul edilen geleneksel İleri Yönlü YSA topolojisini temel almaktadır. İlk olarak seçilen veri seti için en iyi performansa sahip İleri yönlü 
YSA modeli düzenli ağ olarak kabul edilir, daha sonra düzenli ağ topolojisinde, Newman-Watts bağlantı yenileme algoritması [12] kullanılarak aralarında bağlantı bulunmayan nöron çiftleri rastgele belirlenerek, bu nöron çiftleri arasında yeni bağlantılar oluşturulur. Yeni bağlantı ekleme süreci; aynı katman nöronları hariç, arasında bağ bulunmayan nöron çiftlerinin sayısı ile belirlenen olası maksimum bağlantı sayısı kadar devam ettirilip, her yeni bağlantı eklendiğinde yeni ăg topolojileri elde edilir. Son olarak elde edilen topolojilerin küçük-dünya ağı olup olmadığının tespiti için kümeleme katsayısı (C) ve karakteristik en kısa yol uzunluğu(L) olarak isimlendirilen iki parametreye eşdeğer kabul edilen Simard ve arkadaşları [15] tarafindan önerilen ve Erkaymaz ve arkadaşları [4,16], tarafından kapsamı geliştirilen, lokal katsayı ( $\mathrm{D}_{\text {Lokal }}$ ) ve global katsayıları ( $\left.D_{\text {Global }}\right)$ kullanılmıştır. Burada; $D_{\text {Lokal }}, 1 / C$ ile eşdeğer, $D_{\text {Global }}$ ise $L$ parametresine eşdeğer kabul edilmektedir. Her iki katsayının da minimum değerde olduğu aralıkta ağın küçük-dünya ağı olduğu kabul edilmektedir [4,15-16,21]. Bu süreçte; Global katsayı aşağıdaki gibi tanımlanmaktadır [4,15$16,20]$.

$$
\begin{gathered}
\mathrm{D}_{\text {Global }}=\frac{1}{\mathrm{E}_{\text {Global }}} \\
\mathrm{E}_{\text {Global }}=\frac{1}{\mathrm{~N}(\mathrm{~N}-1)} \sum_{\mathrm{i} \neq \mathrm{j} \in \mathrm{N}}^{\mathrm{N}} \frac{1}{\mathrm{~d}_{\mathrm{ij}}}
\end{gathered}
$$

Denklemde, $\mathrm{d}_{\mathrm{ij}}$, düğümler arası en kısa yol uzunluğunu, $\mathrm{N}$ ise ağdaki düğüm sayısını ifade etmektedir. Diğer taraftan Lokal katsayı ise aşağıdaki gibi tanımlanmaktadır [4,15-16].

$$
\begin{gathered}
\mathrm{D}_{\text {Lokal }}=\frac{1}{\mathrm{E}_{\text {Lokal }}} \\
\mathrm{E}_{\text {Lokal }}=\frac{1}{\mathrm{~N}} \sum_{\mathrm{i}=1}^{\mathrm{N}} \mathrm{E}\left(\mathrm{G}_{\mathrm{i}}\right) \\
\mathrm{E}\left(\mathrm{G}_{\mathrm{i}}\right)=\frac{1}{\mathrm{~N}(\mathrm{~N}-1)} \sum_{\mathrm{k} \neq \mathrm{l} \in \mathrm{N}}^{\mathrm{N}} \frac{1}{\mathrm{~d}_{\mathrm{kl}}}
\end{gathered}
$$

Denklemde, $G_{i}$, i. Dügüm çıkarıldığında geriye kalan çizgeyi, $\mathrm{N}_{\mathrm{i}}$, sadece i. düğüme ait komşu düğüm sayısını, $d_{k l}$, ise $i$. düğüm çıkarıldığında geriye kalan çizgenin dügü̈mleri arası en kısa yol uzunluğunu ifade etmektedir.

Elde edilen ağ topolojilerinin öğrenme performanslarını test edebilmek için, Rprop algoritması ağ modeline uyarlanmıştır. Ek olarak her nöronun aktivasyon fonksiyonu ise bipolar-sigmoid olarak tanımlanmıştır. Rprop algoritması geleneksel geriye yayılım algoritmasından türetilen bir algoritma olmakla birlikte, geri yayılım algoritmasından farklı olarak hata eğiminin genliği yerine, eğimin işaretini kullanarak güncelleme yönünü belirlemektedir. Buna ek olarak, Rprop algoritması her ağırlık için adım sayısının dinamik olarak değiştirerek ağırlıkları farklı değerlerle değiştirir. Böylece öğrenme sürecinde bir adaptasyon ve hız sağlanmaktadır. Sonuç olarak, hatanın kısmi türevinden kaynaklı eğim sorunu elimine edilmektedir. Rprop algoritmasında ağırlıklar ve ağırlıklardaki değişim aşağıdaki gibi tanımlanır:

$$
\Delta \mathrm{w}_{\mathrm{ij}}(\mathrm{t})=\left\{\begin{array}{c}
\min \left(\mathrm{l}^{+*} \Delta_{\mathrm{ij}}(\mathrm{t}-1), \Delta_{\max }\right), \text { if } \frac{\partial \mathrm{E}}{\partial \mathrm{w}_{\mathrm{ij}}}(\mathrm{t}-1) * \frac{\partial \mathrm{E}}{\partial \mathrm{w}_{\mathrm{ij}}}(\mathrm{t})>0 \\
\max \left(\mathrm{l}^{-*} \Delta_{\mathrm{ij}}(\mathrm{t}-1), \Delta_{\min }\right), \text { if } \frac{\partial \mathrm{E}}{\partial \mathrm{w}_{\mathrm{ij}}}(\mathrm{t}-1) * \frac{\partial \mathrm{E}}{\partial \mathrm{w}_{\mathrm{ij}}}(\mathrm{t})<0 \\
\Delta_{\mathrm{ij}}(\mathrm{t}-1), \text { otherwise }
\end{array}\right\}
$$


Denklemde, $1\left(\mathrm{l}^{+}>1>\mathrm{l}^{-}>0\right)$, işlem faktörüdür, Modelde bu değer sırasıyla bu değer, $\mathrm{l}^{+}=1.2$ ve $1^{-}$ $=0.5$ olarak belirlenmiştir. $E$, hata fonksiyonunu, $w_{i j}$ is $i$. ve j. nöronlar arası ağırlığı ve $\Delta w_{i j}$ ağırlıktaki değişimi ifade etmektedir. Eğitim süreci, 10-kat çapraz doğrulama yöntemi kullanılarak gerçekleştirilmektedir. Maliyet fonksiyonu olarak Ortalama karesel hata (MSE) kullanılmıştır. Eğitilen ağlar, MSE parametresinin yanında, Ortalama mutlak hata (MAE), determinasyon katsayısı $\left(R^{2}\right)$ yardımıyla istatistiksel olarak test edilmektedir. Denklemlerde; $\mathrm{N}$, örnek sayısı, $\mathrm{y}_{\mathrm{x}}$, gerçek değeri, $\mathrm{p} x$, tahmin edilen değeri ifade etmektedir.

$$
\begin{aligned}
& \mathrm{MSE}=\frac{1}{2 \mathrm{~N}} \sum_{\mathrm{x}=1}^{\mathrm{N}}\left(\mathrm{y}_{\mathrm{x}}-\mathrm{p}_{\mathrm{x}}\right) \\
& \mathrm{MAE}=\frac{1}{\mathrm{~N}} \sum_{\mathrm{x}=1}^{\mathrm{N}}\left|\mathrm{y}_{\mathrm{x}}-\mathrm{p}_{\mathrm{x}}\right| \\
& \mathrm{R}^{2}=1-\frac{\sum_{\mathrm{x}=1}^{\mathrm{N}}\left(\mathrm{y}_{\mathrm{x}}-\mathrm{p}_{\mathrm{x}}\right)^{2}}{\sum_{\mathrm{x}=1}^{\mathrm{N}} \mathrm{p}_{\mathrm{x}}^{2}}
\end{aligned}
$$

\section{Bulgular ve Tartışma}

$\mathrm{Bu}$ çalışmada, Esnek küçük-dünya ağ modelinde çok katmanlı topolojik etkinin sonuçlarını gözlemek için sentetik fonksiyon yaklaşımı problemi oluşturulmuş ve hazırlanan veri seti ile performans ölçümü yapılmıştır. Bu süreçte, öncelikle İleri yönlü YSA topolojisi belirlenip düzenli ağ olarak kabul edilir. Düzenli ağ topolojilerine Newman-Watts yeni bağlantı ekleme yaklaşımı kullanılarak farklı topolojiler elde edilir. $\mathrm{Bu}$ mimarilere Esnek geri yayılım algoritması adapte edilerek Esnek küçük-dünya ağları oluşturulmaktadır. Son olarak ise belirlenen veri setiyle performans analizleri yapılmıştır.

Elde edilen modellerin testini gerçekleştirmek amacıyla sinüzoidal bir fonksiyon yaklaşım problemi seçildi: $F_{x}=\prod_{x=0}^{1} x^{2} \sin (5 \pi x)^{3}$ Hazırlanan fonksiyon Şekil 1'de gösterilmiştir.

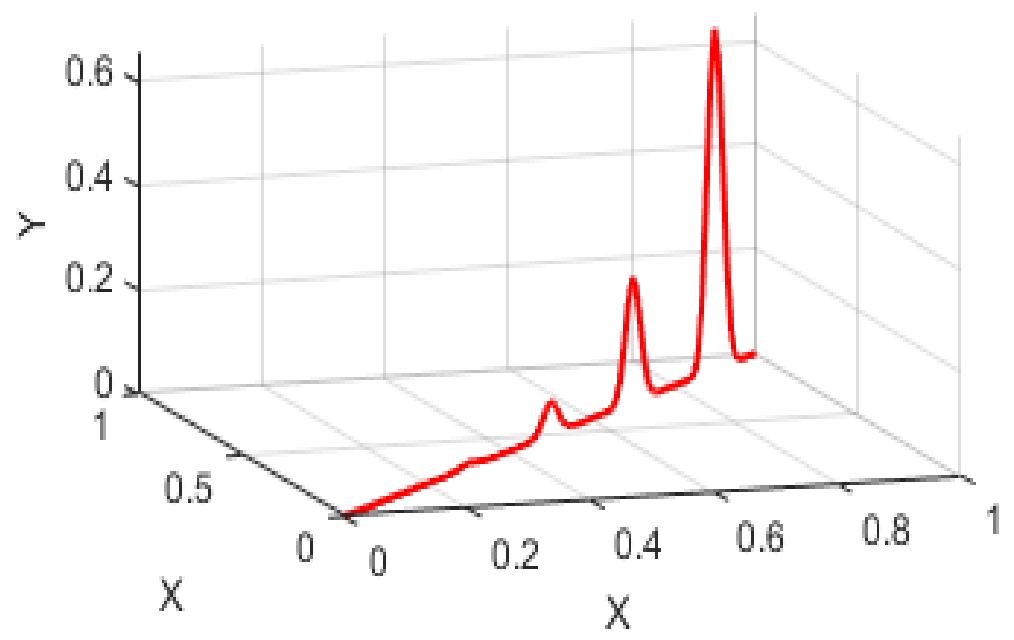

Şekil 1. $\mathrm{F}_{\mathrm{x}}$ Fonksiyon yaklaşım problemi.

Metodolojinin başlangıç aşamasında, geleneksel YSA modelini belirlemek için gizli katman deneyi gerçekleştirilmekte ve temel düzenli ağ topolojileri elde edilmektedir. Bu bağlamda, İleri yönlü YSA topolojisi sırasıyla bir, iki ve üç gizli katmana sahip olacak şekilde (2-H-1), (2-H-H-1) ve (2$\mathrm{H}-\mathrm{H}-\mathrm{H}-1)$ mimarili olarak seçilmiştir. Bu ifadelerde $\mathrm{H}$ gizli katman nöron sayısını göstermektedir. Seçilen topolojilerin gizli katman nöron sayısını bulmak amacıyla; $\mathrm{H}=2: 2: 14$ olarak alınmış ve sabit başlangıç ağırlıkları kullanılarak gizli katman deneyi benzetimleri gerçekleştirilmiştir. $\mathrm{Bu}$ işlem sonucunda; bir gizli katmanlı topoloji için en iyi hata değeri $\mathrm{H}=12$ olarak bulunmuş ve ağ topoloji 
(2-12-1) olacak şekilde tasarlanmıştır. İki gizli katmanlı topoloji için en iyi hata değeri $\mathrm{H}=4$ ve topoloji (2-4-4-1) olacak şekilde elde edilmiştir. Son olarak üç gizli katmanlı topoloji için en iyi hata değeri $\mathrm{H}=4$ bulunmuş ve topoloji (2-4-4-4-1) olacak şekilde belirlenmiştir. Topolojiler belirlendikten sonra, ikinci aşamada, yenilenebilir bağlantı sayısı (YBS) değeri her bir topoloji için hesaplanmıştır. YBS değeri farklı katmanlardaki bağlı olmayan nöron çiftleri sayısını ifade etmektedir. Bu bağlamda, bu değer; bir gizli katmanlı 2-12-1 topolojisi için YBS=2, iki gizli katmanlı 2-4-4-1 topolojisi için YBS=6 ve üç gizli katmanlı 2-4-4-4-1 topolojisi için YBS=40 olarak hesaplanmıştır. Bir sonraki adımda, her bir topoloji için YBS değeri kadar Newman-Watts bağlantı yenileme prosedürü uygulanmış ve elde edilen topolojiler için global-lokal katsayılar hesaplanmıştır. Tek katmanlı mimaride sadece giriş ve çıkış katmanı nöronları arasında bağlantı ekleme prosedürü uygulandığı için küçük-dünya ağı davranışı, global ve lokal katsayının minimum olduğu yenilenen bağlantı $(\mathrm{Rw})$ değerinin 1-2 arasında olması gerektiği gözlemlenmiştir. Bu nenle, $\mathrm{Rw}=0$ iken geleneksel Esnek ileri yönlü YSA ağı kabul edilirken, diğer durumda $(\mathrm{Rw}=1-2)$ Ağ Esnek küçük-dünya ağı olarak kabul edilmektedir. Son olarak elde edilen mimariler Şekil 1'de belirlenen yaklaşım fonksiyonu problemi veri seti ve 10-kat çapraz doğrulama metodu ile eğitilerek test sonuçları Tablo 1'de verilmiştir.

Tablo 1. Fonksiyon yaklaşımı problemi 2-12-1 mimarisi test performans sonucu.

\begin{tabular}{cccccc}
\hline YBS & Mse & Mae & $\mathbf{R}^{\mathbf{2}}$ & D $_{\text {Global }}$ & D $_{\text {Lokal }}$ \\
\hline $\mathrm{Rw}=0$ & 0.021641 & 0.071000 & 0.394720 & 5.675 & $\infty$ \\
\hline $\mathbf{R w}=\mathbf{1}$ & $\mathbf{0 . 0 0 4 6 9 0}$ & $\mathbf{0 . 0 3 2 8 0 0}$ & $\mathbf{0 . 7 8 3 3 9 9}$ & $\mathbf{5 . 6}$ & $\mathbf{1 9 5}$ \\
\hline $\mathrm{Rw}=2$ & 0.004843 & 0.033074 & 0.781180 & 5.3 & 98 \\
\hline
\end{tabular}

Görüldüğü üzere (Tablo 1), düzenli ağa göre $\mathrm{Rw}=1$ ile elde edilen topoloji en iyi test performans1 sergilemiştir. Benzer şekilde, bir katmanlı mimari için uygulanan bağlantı yenileme süreci iki gizli katmanlı 2-4-4-1 topolojisi için de uygulanmış ve elde edilen mimariler için global-lokal katsayı hesaplanarak sonuçlar Şekil 2'de gösterilmiştir.

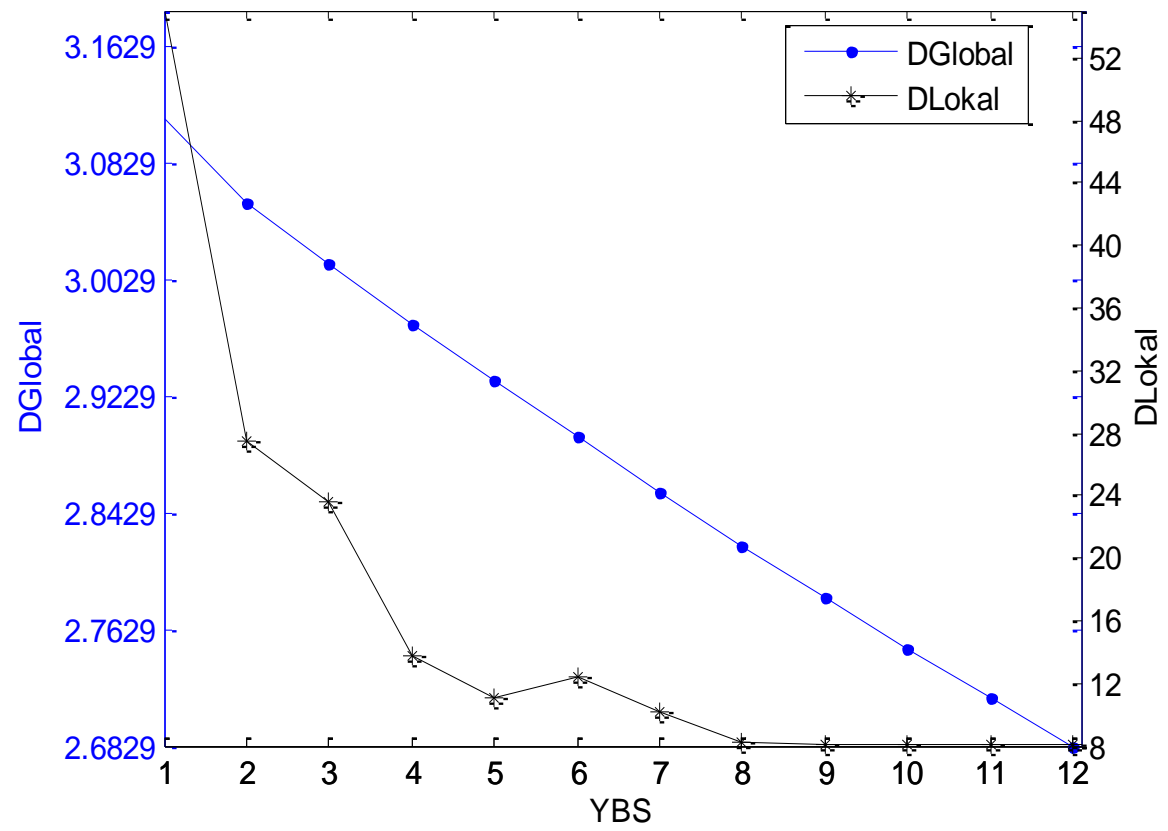

Şekil 2. 2-4-4-1 mimarisi bağlantı yenileme süreci sonucu, $D_{\text {Global }}$ ve $D_{\text {Lokal }}$ değişimi.

Şekil 2'de görüldüğü gibi, RW=4 değeriyle birlikte global ve lokal katsayıların eğimlerindeki değişimim azalarak minimize olmaktadır. Bu nedenle, küçük-dünya davranışı $\mathrm{Rw}=4-12$ arasında 
gözlemlenebilmektedir. Gözlemlenen ağ topolojileri, tanımlanan veri setiyle eğitilmiş ve test analiz sonuçları Tablo 2'de gösterilmiştir.

Tablo 2. Fonksiyon yaklaşımı problemi 2-4-4-1 mimarisi test performans sonucu.

\begin{tabular}{cccc}
\hline YBS & Mse & Mae & $\mathbf{R}^{\mathbf{2}}$ \\
\hline $\mathrm{Rw}=0$ & 0.018824 & 0.076872 & 0.153636 \\
$\mathrm{Rw}=1$ & 0.004289 & 0.039454 & 0.807153 \\
$\mathrm{Rw}=2$ & 0.029432 & 0.066232 & 0.365857 \\
$\mathrm{Rw}=3$ & 0.029431 & 0.066110 & 0.366393 \\
$\mathrm{Rw}=4$ & 0.012113 & 0.046560 & 0.455378 \\
$\mathrm{Rw}=5$ & 0.008005 & 0.039115 & 0.640105 \\
$\mathrm{Rw}=6$ & 0.015179 & 0.051684 & 0.317523 \\
$\mathrm{Rw}=7$ & 0.003240 & 0.025719 & 0.854308 \\
$\mathrm{Rw}=8$ & 0.003472 & 0.026937 & 0.843911 \\
$\mathrm{Rw}=9$ & 0.003164 & 0.025261 & 0.857744 \\
$\mathrm{Rw}=10$ & 0.003566 & 0.028299 & 0.839685 \\
\hline $\mathrm{Rw}=\mathbf{1 1}$ & $\mathbf{0 . 0 0 3 0 3 0}$ & $\mathbf{0 . 0 2 4 1 0 4}$ & $\mathbf{0 . 8 6 3 7 4 8}$ \\
\hline $\mathrm{Rw}=12$ & 0.003210 & 0.026173 & 0.855694
\end{tabular}

Tablo 2'de görüldüğü gibi, Şekil2'de gözlemlenen aralıkta topolojilerde performans artış1 gözlemlenmiş ve $\mathrm{Rw}=11$ için en iyi Esnek küçük-dünya ağı performansı elde edilmiştir. $\mathrm{Bu}$ topolojinin performansının Geleneksel Esnek topolojiye göre(RW=0) \%57 daha iyi olduğu görülmüştür. Buradan hareketle, katman sayısının artırılmasının olumlu etki gösterebildiği deney imlenmiştir. Son olarak bağlantı yenileme süreci üç katmanlı 2-4-4-4-1 mimariye uygulanmış ve global-lokal katsayılar hesaplanmıştır. Elde edilen sonuçlar Şekil 3'te gösterilmiştir.

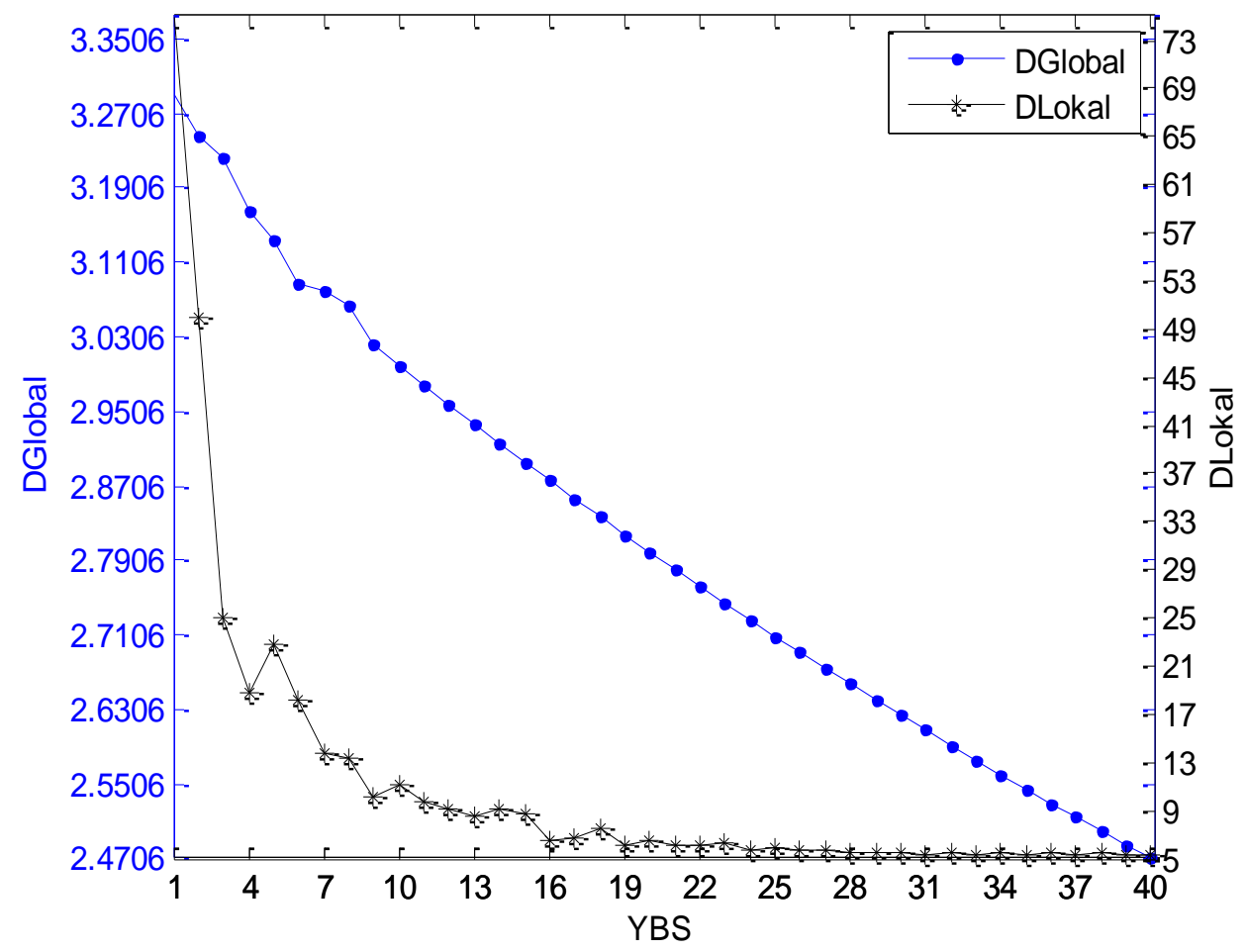

Şekil 3. 2-4-4-4-1 mimarisi bağlant1 yenileme süreci sonucu, $D_{\text {Global }}$ ve $D_{\text {Lokal }}$ parametreleri değişimi. 
Şekil 3'te görüldüğü gibi diğer topolojilere benzer şekilde global-lokal katsayının eğimindeki değişim $\mathrm{Rw}=8-40$ arasında azalarak minimum değerlerine ulaşmakta ve bu aralıkta küçük-dünya ağı gözlemlenmektedir. Elde edilen tüm topolojilere benzer başlangıç koşullarında, tüm eğitim ve analiz süreci uygulanmış ve sonuçlar Tablo 3 ’te gösterilmiştir.

Tablo 3. Fonksiyon yaklaşımı problemi 2-4-4-4-1 mimarisi test performans sonucu.

\begin{tabular}{|c|c|c|c|}
\hline YBS & Mse & Mae & R2 \\
\hline $\mathrm{Rw}=0$ & 0.014644 & 0.065333 & 0.407357 \\
\hline $\mathrm{Rw}=2$ & 0.024065 & 0.053147 & 0.456192 \\
\hline $\mathrm{Rw}=4$ & 0.003107 & 0.025122 & 0.862660 \\
\hline $\mathrm{Rw}=6$ & 0.028964 & 0.066087 & 0.366992 \\
\hline $\mathrm{Rw}=8$ & 0.003037 & 0.024400 & 0.863724 \\
\hline $\mathrm{Rw}=10$ & 0.003570 & 0.028227 & 0.847161 \\
\hline $\mathrm{Rw}=12$ & 0.004758 & 0.031600 & 0.789477 \\
\hline $\mathrm{Rw}=14$ & 0.025768 & 0.051407 & 0.456528 \\
\hline $\mathrm{Rw}=16$ & 0.002531 & 0.017155 & 0.907045 \\
\hline $\mathrm{Rw}=18$ & 0.004633 & 0.030457 & 0.793237 \\
\hline $\mathrm{Rw}=20$ & 0.028320 & 0.065786 & 0.368604 \\
\hline $\mathrm{Rw}=22$ & 0.026338 & 0.051160 & 0.453401 \\
\hline $\mathrm{Rw}=24$ & 0.003104 & 0.024230 & 0.858876 \\
\hline $\mathrm{Rw}=26$ & 0.016890 & 0.051901 & 0.503779 \\
\hline $\mathrm{Rw}=28$ & 0.028901 & 0.066189 & 0.364609 \\
\hline $\mathrm{Rw}=30$ & 0.011680 & 0.045211 & 0.602539 \\
\hline$R w=31$ & 0.000199 & 0.006195 & 0.990486 \\
\hline $\mathrm{Rw}=32$ & 0.029449 & 0.066122 & 0.369150 \\
\hline $\mathrm{Rw}=34$ & 0.003045 & 0.024458 & 0.863484 \\
\hline $\mathrm{Rw}=36$ & 0.000731 & 0.016485 & 0.963673 \\
\hline $\mathrm{Rw}=38$ & 0.021691 & 0.071023 & 0.394523 \\
\hline $\mathrm{Rw}=40$ & 0.006806 & 0.038092 & 0.713970 \\
\hline
\end{tabular}

Tablo 3'te görüldüğü gibi Şekil2'de gözlemlenen aralıta $\mathrm{Rw}=31$ için en iyi performansa sahip Esnek küçük-dünya ağı gözlemlenmiştir. Geleneksel Esnek topolojiye göre $(\mathrm{RW}=0)$ performansın yaklaşık \%150 arttı̆̆ görülmüştür.

\section{Sonuç ve Öneriler}

Deterministik olarak kestirimi zor fonksiyon yaklaşım(regresyon) problemleri için geleneksel yapay zekâ araçlarında, yeni bir yaklaşım geliştirmek önemli bir araştırma konusudur. Bu bağlamda, bu çalışmada Erkaymaz [20] tarafından önerilen Esnek küçük-dünya YSA modeli örnek bir regresyon problemine uygulanmış ve YSA araştırmalarında en önemli sorun olan topolojik tasarım problemi araştırılmıştır. $\mathrm{Bu}$ amaç için çok katmanlı Esnek küçük-dünya ağ topolojileri elde edilmiş ve analizler gerçekleştirilmiştir. Bu topolojiler bir, iki, üç katmanlı ve 2-14 arasında değişen gizli katman nörona sahip olacak şekilde tasarlanmış ve belirlenen problem veri setiyle eğitilip istatistik testlere tabi tutulmuşlardır. Sonuçlar incelendiğinde, tek katmanlı (2-12-1) YSA topolojisini temel alan en iyi Esnek küçük-dünya ağ topolojisi $(\mathrm{Rw}=1)$ performans değerleri; Mse=0.00469, R2=0.7834 olarak elde edilmiş, İki katmanlı (2-4-4-1) YSA topolojisini temel alan en iyi Esnek küçük-dünya ağ topolojisi $(\mathrm{Rw}=11)$ performans değerleri; Mse=0.003030, $\mathrm{R} 2=0.8637$ olarak gözlemlenmiştir. Son olarak ise üç katmanlı (2-4-4-4-1) YSA topolojisini temel alan en iyi Esnek küçük-dünya ağ topolojisi $(\mathrm{Rw}=31)$ performans değerleri; Mse $=0.000199, \mathrm{R} 2=0.99048$ olarak elde 
edilmiştir. Sonuç olarak, fonksiyon yaklaşımı probleminde, önerilen esnek küçük-dünya YSA modeli geleneksel Esnek YSA modeline göre performansı geliştirmektedir. Aynı benzetim koşulları için katman sayıları için performans \%50-150 arasında artmaktadır. Diğer taraftan, katman sayısı artarken, gizli katman nöron sayısının artırılmasının performansı negatif etkilediği görülmüştür. Bu etkinin artan nöron sayısından kaynaklı algoritmanın zaman karmaşıklığının artmasından kaynaklandığı öngörülmüştür. Bu bilgi ışı̆̆ında söylenebilir ki, katman sayısı artırıldığında en iyi ağ performansları düşük gizli katman nöron sayılarında elde edilmektedir. Toplamda, bu yaklaşım gelecekte derin nöron ağları performansının geliştirilmesinde anahtar rol oynayabileceği öngörülmüştür. Gelecek çalışmalarımızda bu konu üzerine araştırmalar yapılacaktır.

\section{Kaynaklar}

[1]. Haykin, S., Neural networks - a comprehensive foundation, (2nd Edition), Prentice-Hall, Englewood Cliffs, NJ., 1999.

[2]. Magnitskii N A., Some New Approaches to the Construction and Learning of Artificial neural Networks, Computational Mathematics and Modeling, 2001,12, 293-304.

[3]. Sun, M., Stam, A., Steuer, RE., Solving Multiple Objective Programming Problems Using Feed-Forward Artificial Neural Networks: The Interactive FFANN Procedure, Man. Sci., 1996, 42(6), 835-849.

[4]. Erkaymaz, O., Ozer, M \& Yumusak, N., Impact of small-world topology on the performance of a feed-forward artificial neural network based on 2 different real-life problems, Turk. J. Elec. Eng. \& Comp. Sci., 2014, 22, 708-718.

[5]. Riedmiller, M. and Braun, H., A direct adaptive method for faster backpropagation learning: The RPROP Algorithm, Proceedings of the IEEE International Conference on Neural Networks. 1993, 586-591.

[6]. Riedmiller, M., Braun, H., Neural speed controller trained online by means of modified rprop algorithm, IEEE Trans. Ind. Inform, 2015, 11, 586-591.

[7]. Shrestha, S. B. \& Song, Q., Robust learning in SpikeProp. Neural Networks, 2017, 86, 54-68.

[8]. Yilmaz, E., Baysal, V., Ozer, M., Perc, M. Autaptic pacemaker mediated propagation of weak rhythmic activity across small-world neuronal networks, Phys. A, 2016, 444, 538-546.

[9]. Yilmaz, E., Baysal, V., Perc, M., Ozer, M., Enhancement of pacemaker induced stochastic resonance by an autapse in a scale-free neuronal network, Sci. China Tech. Sci., 2016, 59(3),364-370.

[10]. Watts, D. J. \& Strogatz, S. H., Collective dynamics of 'small-world' networks, Nature, 1999, 393, 409-10.

[11]. Watts D. J., Small Worlds: The Dynamics of networks between order and randomness, Princeton, NJ:Princeton University Press, 2003.

[12]. Newman, M. E. J. \& Watts, D. J., Scaling and percolation in the small-world network model, Phys. Rev. E, 1999, 60, 7332-7342.

[13]. Kawai, Y., Park, J. \& Asada, M., A small-world topology enhances the echo state property and signal propagation in reservoir computing, Neural Networks, 2019, 112, 15-23.

[14]. Barabasi, AL., Albert, R., Emergence of Scaling in Random Networks, Science, 1999, 286, 509-512.

[15]. Simard, D., Nadeau, L. \& Kröger, H., Fastest learning in small-world neural networks, Physics Letters A., 2005, 336(1),8-15.

[16]. Shuzhong, Y., Siwei, L., Jianyu, Li., Building Multi-layer Small World Neural Network, Lect. Note in Comp. Sci. Ser., 2006, 3971, 695-700.

[17]. Erkaymaz, O., Ozer, M., Impact of small-world network topology on the conventional artificial neural network for the diagnosis of diabetes. Chaos Sol. and Fract., 2016, 83, 178185. 
[18]. Erkaymaz, O., Ozer, M., Impact of Newman-Watts Small-World approach on The Performance of Feed-Forward Artificial Neural Networks, Karaelmas Science and Engineering Journal., 2016, 6(1), 187-194.

[19]. Erkaymaz, O., Ozer, M. \& Perc, M., Performance of small-world feedforward neural networks for the diagnosis of diabetes, Appl. Math Comput., 2017, 311, 22-28.

[20]. Erkaymaz, O., Resilient back-propagation approach in small-world feed-forward neural network topology based on Newman-Watts algorithm, Neural Comput \& Applic., 2020, https://doi.org/10.1007/s00521-020-05161-6.

[21]. Latora, V. \& Marchiori, M. (2001). Efficient behavior of small-world networks, Phys. Rev. Letter., 2001, 87(19), 98701. 